\title{
The Effect of Linguistic Theory on Students' Intelligence Cultivation in Chinese Teaching Practice
}

\author{
Wang Sha, Xiao Jiugen* \\ Jiangxi Normal University, Research Center of Language and Language Life, Nanchang, China
}

Email address:

756987919@qq.com (Wang Sha),jxsdxjg666666@sina.com (Xiao Jiugen)

${ }^{*}$ Corresponding author

\section{To cite this article:}

Wang Sha, Xiao Jiugen. The Effect of Linguistic Theory on Students' Intelligence Cultivation in Chinese Teaching Practice. Science Innovation. Vol. 7, No. 4, 2019, pp. 125-128. doi: 10.11648/j.si.20190704.14

Received: September 21, 2019; Accepted: November 4, 2019; Published: November 8, 2019

\begin{abstract}
Linguistic theory comes from linguistic practice, which is the only source to enrich linguistic theory. Vice versa, language practice cannot be separated from the guidance of linguistic theory. Once leaving linguistic theory, its language practice will lose more than it gains and even cause serious losses, which is fully proved by language teaching practice. In recent years, Chinese teaching has abandoned the guidance of linguistic theories, resulting in unsatisfactory teaching results and certain irreparable losses. It is based on this point that, when reviewing the current situation of Chinese teaching, the author puts forward that the linguistic theory should not only be used to guide the practice of Chinese teaching, but also be used to solve the problems related to Chinese language teaching. On the premise of improving students' thinking ability and intelligence level, efforts should be made to improve their language ability.
\end{abstract}

Keywords: Linguistic Theory, Chinese Teaching, Intelligence Cultivation, Language Ability, Measures Suggested

\section{语言学理论在语文教学实践中对学生智能培养的作用}

\section{王莎, 肖九根 ${ }^{*}$}

江西师范大学语言与语言生活中心, 南昌, 中国

\section{邮箱}

756987919@qq.com（王莎）, jxsdxjg666666@sina.com（肖九根）

摘要: 语言学理论来源于语言实践, 语言实践是丰富语言学理论的唯一源泉; 反之亦然, 语言实践离不开语言学 理论的指导, 一旦离开语言学理论, 其语言实践就得不偿失, 甚至会造成严重损失, 语文教学实践就充分证明了 这一点。近些年来, 语文教学抛开了语言学理论的指导, 致使教学效果很不理想, 造成了一定的无法弥补的损失。 正是基于这点, 在审视语文教学现状的当下, 提出了不仅要将语言学理论运用于指导语文教学实践, 还要用之于 解决与语文教学中存在的系列相关理论与实践问题, 要在提高学生的思维能力、智能水平前提下, 努力地提高他 们的语言能力。

关键词: 语言学理论, 语文教学, 智能培养, 语言能力, 建议措施 


\section{1. 引言}

一般地说, 高等师范院校的主要任务是为基础教育培 养师资的, 其课程的设置应为基础教育服务; 基础教育也 应以师范院校的课程理论为指导。但实际情况并非如此。 长期以来, 师范院校课程的开设与教学在某种程度上同基 础教育存在严重脱节的现象, 没有对基础教育起到应有的 指导作用, 而基础教育也没有自觉地以师范院校课程理论 作指导, 以之解决基础教育中的实际问题, 如师范院校的 语言学理论课与基础语文教学就存在类似情况。有学者认 为: “中国语文教育的问题, 很大程度上源于其语言学基 础落后和语用型语文知识缺失。”[1]语文教育效率低下的 问题, 早已引起了专家学者的关注。不仅如此, 他们还就 基础语文教育如何提高教学效果问题提出了自己的真知 灼见。例如, 上世纪末, 张志公先生 (1994) 在谈到语言 学理论与语文教学的关系时, 就提出了要在语言学基础知 识、基础理论和语文教学之间建立一种桥梁性学科的构想 [2]（第302页）。王宁先生（2000）一贯认为语言学对语 文教学有着十分重要的指导作用, 因为“有了语言学的指 导, 语文教师的教学工作会自觉起来, 办法会灵活起来, 程序会合理起来, 效果的检验也将成为可能”[3]。苏宝荣 先生 (2002) 还着重阐述了语言学理论对提高语文教学质 量的重要性问题[4]。此外, 郑振峰（2009）[5]、倪宝元 （1998）[6]、王建华（2000）[7]等学者对这一问题也作 了较为深入的分析, 并为解决其症结问题出计献策。本文 论述的着眼点虽在于此, 但主要就语言学理论运用于语文 教学实践中, 如何在学生智能培养方面发挥它应有的作用 作些探讨, 这是学者们至今尚未论及过的。毫无疑问, 语 言学理论对学生的智能培养是有重要作用的。

\section{2. 学校的语言教育是促使学生智能形成的一条 基本途径}

语言学理论认为, 语言是人的智能活动必不可少的要 素, 是人们摄取外部信息进行信息加工不可或缺的手段。 对青少年进行语言教育, 实质上就是对其思维进行优化, 这是促使他们智能形成的一个最重要的手段, 也是一条行 之有效的基本途径。

语文教学是语言教育的实践活动, 也是培养青少年智 能的一项基本而重要的活动。在基础语文教学中, 一个教 师如能运用语言学基础理论, 自觉地把语言训练和思维培 养结合起来, 这对青少年智能的形成是大有禆益的。对于 语文教育科学的研究, 有学者主张: “今后教育科学的研 究方向, ...... 一定要坚持理论与实际相结合。”[8]其“理论” 就是语言理论知识, “实际”则是语文教学实践。只有将语 言理论知识与语文教学实际结合起来, 语文教育才能更科 学, 出成效。

教育心理学原理告诉我们: “智力技能是借助内部语 言在人脑内部进行的认识方式, 是以思维为核心的认识加 工的具体方式。......智力活动是靠内部语言及词的作用进 行的, 是以词的形式在“心里”完成的动作。”[9] (第194页) 其“内部语言及词”亦谓语言理论知识在人脑中的映像, 这 些人脑中储存的映像给智力活动提供“能量来源”。而“能
量源”又与人的智力活动源成正比关系。也就是说, 如“能 量源”贫乏, 那智力活动源也贫乏; 反之, 其智力活动源 就丰富。由此可见, 熟练掌握和运用语言理论知识, 对于 人的智能培养有着十分重要的作用。

在智能培养有关研究中, 国内外心理学家一致公认思 维是智能的核心。思维能力的培养, 能使人的智能得到迅 速而有效的发展。

尽管是语言决定思维, 还是思维决定语言, 是语言学 中至今最具争议的问题之一 $[10]$, 但是不论谁决定谁, 人 们都可以看出语言与思维二者之间的密切关系。德国著名 语言学家洪堡特在《论人类语言结构的差异及其对人类精 神的影响》里说到: “语言是构成思想的器官。智力活动 完全是精神的和内在的, 一定程度上会不留痕迹地逝去, 这种活动通过声音而在言语中得到外部表现, 并为感官知 觉到。因此, 智力活动与语言是一个不可分割的整体。”[11]

(第65页) 不论独自冥想还是与人交流, 人们都要通过语 言进行思维, 这是不容否认的。因为语言不仅是人类最重 要的交际工具, 还“是思维的工具, 也是认识成果的咜存 所”[12]（第14页）。思维过程离不开语言, 需要借助语言 进行比较、分析、推理、综合等一系列活动, 也需要借助 语言形成思想, 并把它储存于大脑之中, 进而将其表达出 来, 传达给人。而且, 人类思维的成果, 必须通过语言中 的词语、句子才能固定下来。也就是说, 思维活动离不开 语言, 必须借助语言材料才能进行。同样, 语言也离不开 思维, 二者相互依存, 共同发展。一句话, 思维是智能的 核心, 语言是思维的载体。要使人的智能得以形成, 就应 提高思维能力; 而要提高思维能力, 就应提高语言能力。 所以, 一个人语言能力的提高能促使他的思维发展, 从而 有助于其智能的形成。王安石有句名诗: “春风又绿江南 岸。”诗中的“绿”字是在改了“到”“过”“入”“满”等字之后才 最后确定的。此处的“绿”既直接生动地描绘了江南两岸的 盎然春意, 又间接隐晦地反映了作者仕途的光明前景, 可 谓“一字双雕”。这样, 诗句经过作者千锤百炼, 融多种修 辞艺术于一体, 使之成为千古名句, 显然不是随意而作, 而是倾注了作者的心智所产生的成果。文学家是这样, 那 一般的人 (包括学生) 就更是这样, 只有多琢磨, 勤思考, 不断地提高语言运用能力, 才能极大地优化他的思维方 式, 较快地促进他的智能形成与发展。

\section{3. 教师的语言素养是影响学生智能培养的一个 重要条件}

在语文教学中, 教师这个角色对学生的智能培养是很 重要的, 因为他们不仅是主体, 也是客体, 具有双重身份。 语文课中的言语作品, 教师和学生都要经过阅读、思考的 阶段，才能真正领会它的意义。当教师阅读、思考时，他 们就是作品的接受者, 是客体; 而当他们走上讲台给学生 讲授这些言语作品时, 学生则为作品的接受者, 是客体, 教师已经转换为作品的“代言人”, 其所起的作用就是为学 生与作品之间架起一座连接的桥梁。当然, 教学效果如何, 是由多方面因素决定的, 如教学主导、教学主体、教学内 容、教学方式、教学环境等。在诸多因素之中, 教师的作 
用始终是起至关重要的作用。一个好的教师不仅能姻熟地 驾驭教学内容, 还能依据一定的教学主体 (或教学对象) 以一种恰如其分的教学用语和非常有效的教学手段创造 性地组织课堂教学。这样的教师对其教学内容并非如复读 机一样仅仅满足于“照本宣科”地“复读”, 而是将自己对教 学内容领悟、理解的精华以“自我”的语言进行再创造, 其 内容涉及到字、词、句、段、篇、语法、修辞等语言内容。 诚然, 其教学不论以何种手段, 有一点是不容置疑的, 那 就是离不开语言阐释。正因为这样, 语言对学生的思维训 练和智能培养十分重要。可以这样说, 不论授课内容还是 授课方式, 教师必须运用语言, 也会自觉或不自觉地融入 语言学理论知识。有人说, 在课堂教学中, 教师对知识的 引导和传播起着极为重要的作用, 教师的专业素养和知识 传播手段对学生最终获取语文知识, 提高思维能力和智能 水平都有直接作用和重要影响[13]。大学语文是这样, 而 对教师引导依赖性更强的基础语文教育就更是这样。在语 文教学 (尤其作品教学) 中, 学生语言能力的强弱, 决定 了他们理解欣赏水平的高低。所以, 要提高学生的阅读鉴 赏能力和智能水平, 就要不断地提高他们的语言能力。

在语文教学与学习中, 不少的人往往存在一种偏见: 认为语言学中的语法教学和学习对于提高一个人的语言 能力、思维能力和智能水平没有多大的作用, 其实不然。 近些年来, 由于基础语文完全淡化了语法方面的教学内 容, 结果给语文教育造成了很大损失。不可否认, 基础语 文教育淡化语法教学, 一方面除了对语法的误解外, 另一 方面还是语法教学的某些缺陷、失误造成的。尤其是仅局 限于静止地介绍孤立的语法知识, 缺乏辩证观点所造成的 [6]（第34页）。在基础语文教学中, 有的教师单纯而呆板 地讲解语法理论知识, 结果把它讲“死”了; 如果以灵活实 用的方式讲, 就会产生柳暗花明的效果。所以, 教师应将 语法等语言理论知识与现实生活实例结合起来, 要使学生 感到语言理论知识是与人的生活息息相关的, 这样就会学 得生动有趣、易懂实用, 达到事半功倍的效果。如以课文 《鞠躬尽瘁》中的一个句子为例:

\section{那一年他才 42 岁。}

这里的状语“才”是由副词充当, 表示一种时间关系, 它表达了人们对为人民鞠躬尽瘁的、党的好干部焦裕禄英 年早逝的悲痛惋惜的心情。如果去掉这一状语, 句子就成 了:

\section{那一年他 42 岁。}

句子的语义就变得没有任何思想情感了, 仅是一种纯 客观的叙述，而且对焦裕禄同志的生死不仅漠不关心，甚 至还是冷冰冰的。如果再换另一状语, 其语义或思想情感 也许截然相反。例如:

\section{那一年他已经42岁。}

时间状语“已经”的语义，不仅没有表示说话者的痛惜 之情, 似乎还嫌焦裕禄同志活得太长了。由此可见, 状语 在语义表达方面是有重要意义的, 并非可有可无。如是采 用恰当的方法教学, 就能将枯燥的语法等语言理论知识变 得有血有肉、生动有趣, 而且字里行间还充满着思想情感。 如此一来, 学生不仅学会了语法知识点, 还提高了语言运 用能力, 他们的思维智能也得到了训练。
又如, 语法教学中, 还可以设计这样一个场景: 外面 刮着风, 下着雨, 甲学生坐在窗边。这时, 乙问: “你感 觉冷吗?”丙说: “外面刮风下雨挺冷的。”然后, 让其他 学生猜测乙的言外之意, 要其回答, 由教师点评, 然后因 势利导地阐释陈述句、疑问句的句法结构特点。这样，不 仅会激发学生的兴趣, 他们还会自觉地参与到有关的话题 中来, 这既有利于他们的思维发展, 也有利于他们的智力 开发, 还能活跃课堂气氛, 取得好的教学效果。

不仅如此, 在言语作品教学中, 教师的解读能力也是 很能见其语言功底的。例如, 唐代诗人韦应物《滁州西涧》 中有“野渡无人舟自横”这一诗句, 其表层义不难理解: 郊 野的渡口没见行人，仅有一条小船横漂在水面上。但其深 层义则深刻而丰富: 郊野渡口十分荒凉, 又不见一个人影 就更显得孤寂凄凉, 唯有那独自横漂的小船, 方显一点生 机。诗句寓情于景, 情景交融, 以潮涨春雨中的小舟任自 横泊之景来抒写诗人清闲自怜之情, 实则表达了诗人忧虑 时局而无力请缨的无奈心情; 面对昉狼多如麻的复杂时 局, 诗人没有回天之术, 惟有坦然地闲居于自己的一统世 界, 不“管他冬夏与春秋”了。不容置疑, 一个教师如有较 高的语言素养, 不但能深刻地解读诗意, 还能有意识地将 文学元素与语言元素有机地融为一体。这样一来, 学生的 鉴赏能力一定会得到很大的提高, 其潜在的智能优势也一 定会得到进一步的发展。

\section{4. 基础语文的语境教学是助推学生智能发展的 一种有效方法}

语文教学是离不开语境教学, 而语境教学也就是语用教 学。语境问题或语用问题则是语言学理论中的一个基本问 题。例如, 在言语作品教学中, 无论分析作品语义还是思想 内容, 教师须要联系具体语境中的具体语用内容, 而不能脱 离其具体语境或语用条件去空谈作品的言语意义或思想内 容, 因为言语作品所具有的语义和思想内容是作家在具体语 境或语用条件下所传递的真实而准确的信息。正是基于这一 点, 一个学生如要真正理解作品, 只有将其置于真实的语境 或语用条件下才能得到深入而透彻的领悟, 从而锻炼自己的 思维能力, 发展自己的智力水平。在当今的语文教学实践中, 语用学理论正起着越来越重要的作用, 有的学者甚至把语文 教学就等同于语用教学, 当然这在学界尚存争议, 但语文教 学中融入了一定的语用学理论, 这应该是不容置疑的。在语 文教学中, 如要自然地“获得语文核心素养各个层面的提升 绝非易事, 语文教师只有掌握符合语文学科特性的语言学理 论, 并原创性地运用于培养学生语文核心素养的教学实践, 才能真正走出语文教育的语言之惑” [14], 这正如吕叔湘先生 所说的, 教师“更重要的是通过课堂教学培养学生自己观察 和分析语言现象的习惯, 这不仅有利于他的语文能力的提 高, 也有助于他的智力的开发”[15]（第152页）。诚然, 这 并非短期内所能完成的, 非要长期坚持不可。那么, 如何提 高学生的语言能力, 进一步发展他们的智力水平呢? 语文教 育专家张志公先生认为: “语文课要搞语言训练, 而文学作 品是 “语言的艺术, , 优秀文学作品的语言是丰富的, 运用的 一般是比较精到的, 像曹雪芹的《红楼梦》, 像鲁迅的作品, 
像......还有许多其他作家的作品, 在运用语言方面, 都足以 作为学习的楷模。”[2] (第35页) 所以, 经典作品里的语言 是适应一定的具体语境的, 是语言的典范与精华; 学习经典 作品语境中的语言, 不仅能规范学生的语言, 更为重要的是 能丰富他们的语言, 提高他们的语言能力, 进而发展他们的 智力水平。

事实证明, 联系具体语境 (即各种语场, 无论感性的 还是理性的) 进行语文教学, 这不仅是学生智能形成、发 展的一个重要手段, 还是助推学生智能水平提高的一个加 速器, 因为一个人认知能力强弱与否, 同其智能水平是有 密切关系的。如果其智能水平高, 对事物的认知理解能力 就强; 反之, 认知理解能力就弱。教育心理学研究表明, “智力的材料分为两类: 一类是感性的材料, 另一类是理 性的材料。......理性的材料, 几乎所有研究智力的心理学 家, 都主要强调为语言、数和形。......理性的材料, 主要 是指概念, 不少心理学家称其为符号, 它是用语言对数和 形的各种状态、各种组合和各种特征的概括”[9]（第282 页)。所以说, 在智力理性的材料中, 语言是其重要的组 成部分, 是形成概念必不可少的载体。而概念, 不仅仅存 在于数、形之中, 还存在于每个阶段的学科教学之中, 而 基础语文教学尤为这样。

\section{5. 结语}

就目前的情况而言, 语言学理论与基础语文教学之间 存在着诸多问题。而要解决这些问题, 惟有采取标本兼治 的措施, 方能取得实效。根据这一基本情况, 应从以下几 方面着手解决问题:

第一, 要加强语言学理论对基础语文教学的指导, 重 视用语言理论知识这把钥匙去开启学生的智能大门, 即在 语文教育中强化语言理论知识的启迪与教学。长期以来, 学校的语文教育很大程度上仅重视人文性, 而忽视工具 性, 以致把开启学生智能的有效钥匙—语言理论知识也 给遗弃了, 结果学生不只是语言能力严重下降, 其思维能 力及智能水平也受到了极大的影响, 因此语文教学必须抓 住语言理论知识这个“根本”。

第二, 要通过国培计划等途径, 努力提高语文教师的 语言理论基本素养，惟有加强这个“源”的培育，才能保证 有“源”不断地“流”向语文教育这块实验“地”, 并收获丰硕 果实。严格地说, 当下的语文教师队伍中, 部分教师还难 以承担语文教学这个重任, 因为他们的语言学理论功底十 分薄弱, 有的甚至还处于空白状态。一个教师如果没有系 统而扎实的语言学理论基础, 是不适宜担任语文教学工作 的, 这是有深刻教训的。

第三, 要加快语言学教材的建设步伐, 组织编写具有 师范院校培养语文教育师资特色的教材, 从根本上改变千 校同用一部教材的状况。冊庸讳言, 迄今为止, 师范院校 语言学教材使用的是研究型大学的通用教材, 仅适合培养 研究型人才, 而不具有师范教育实用性强的特点, 更不适 应师范院校培养基础教育师资的实际需要。师范院校的语 言学教材, 应以语文教材作品中的大量言语实例来阐述它 的基本理论与基本规律, 使其真正把语言学理论与基础语
文教学对接起来, 从而保证语言学理论指导语文教学、服 务语文教学的通俗性与实效性。

总的一句话, 语言学理论在语文教学实践中应占有一 席之地, 它不仅可以提高学生的语言能力, 还能提高他们 的思维能力, 进而促使其智能的形成与发展。因此, 作为 基础语文教师, 要根据新时代的发展要求, 熟练掌握语言 学基础理论, 始终以语言学理论指导语文教学实践, 努力 培养出新一代具有较强语言能力、思维能力和智能水平的 实践性基础人才; 作为基础教育的母机一一师范院校, 所 开设的语言学理论课不单要适应培养研究型人才, 更为重 要的是应面向基础教育, 为基础语文教学服务, 为大力培 养实践性教学人才作出应有的贡献。只有这样, 才不偏离 师范院校的办学目标, 不喜负时代赋予的历史使命。

\section{参考文献}

[1] 荣维东, 杜娟.语文教育亟待语用转型与体系重建[J].中国教 育学刊, 2015（05）.

[2] 张志公.语文教育论集[M].人民教育出版社.1994.

[3] 王宁.汉语语言学与语文教学[J].中国社会科学, 2000 (03).

[4] 苏宝荣.语言理论研究与语文教学 [J].河北师范大学学报 (哲 社科版），2002（06）.

[5] 郑振峰, 宋文辉, 李冬鸽.现代语言学和中学教学 [J].河北师 范大学学报（教科版），2009（09）.

[6] 倪宝元. 语言学与语文教育[M].上海教育出版社.1998.

[7] 王建华. 语用学与语文教学[M]. 浙江大学出版社.2000.

[8] Tian Benna.My primary school Chinese education research process [J]. Curriculum,Teaching Material and Method. 2018 (02).

[9] 李 红.教育心理学[M].武汉大学出版社.2007.

[10] Liu Zhicheng.A comparative study of Chinese and western thinking models from the perspective of linguistics [J]. Ideological Line. 2019 (07).

[11] 威廉・冯・洪堡特.论人类语言结构的差异及其对人类精神 的影响[M].商务印书馆.2011.

[12] 叶蜚声, 徐通铭. 语言学纲要 [M].北京大学出版社.1997.

[13] 旷光彩.语言学在大学语文课堂中的地位及应用[J].语文建 设, 2017（10）。

[14] Xu linxiang,Zheng Yun,Re-understanding of "Pragmatic Heat" Based on the Core Competence of Chinese Language [J]. Global Education, 2016 (08).

[15] 吕叔湘.吕叔湘语文论集[A].商务印书馆.1983. 